\title{
Efecto de la suplementación con ensilaje de cáscara de naranja (Citrus sinensis L) sobre algunos parámetros metabólicos en vacas de leche
}

\author{
Effects of the supplementation of with orange (Citrus sinensis $L$ ) peel silage on \\ some metabolic parameters in dairy cows
}

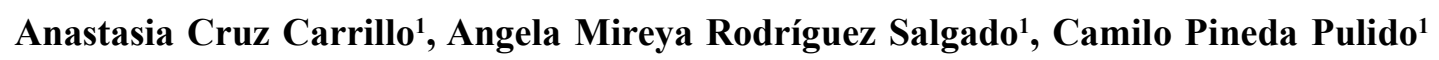

\section{Resumen}

\begin{abstract}
El objetivo de este trabajo fue determinar el efecto del ensilaje de naranja/avena y de solo avena en el comportamiento metabólico de vacas de leche en producción durante la época de verano y sobre la calidad composicional de la leche, buscando alternativas de alimentación para la época de verano sin mayor inversión. Se usaron 20 vacas sanas en producción, divididas en dos grupos al azar. Las vacas del primer grupo se suplementaron con ensilaje de naranja/avena y en el segundo con ensilaje de solo avena por 30 días. Los animales mantuvieron su estado metabólico, condición corporal y producción de leche. El colesterol fue el único parámetro que se encontró más alto en los animales suplementados con ensilaje de naranja/avena. Se concluye que ambos tipos de ensilaje para suplementación son equivalentes, pero la aceptación por parte de los animales del ensilaje de naranja/avena fue notoriamente superior.
\end{abstract}

Palabras clave: bovinos; ensilado; metabolismo; nutrición; alimento

\section{AbSTRACT}

The aim of this study was to determine the effect of orange/oat and only oat silages on the metabolic levels of cows in milk during the summer and on the compositional quality of the milk, looking for alternative feeding during summer without major investments. Twenty healthy cows were distributed in two groups at random. The cows

\footnotetext{
${ }^{1}$ Grupo GIBNA, Programa de Medicina Veterinaria y Zootecnia, Universidad Pedagógica y Tecnológica de Colombia, Tunja, Boyacá, Colombia

${ }^{2}$ E-mail: anastasia.cruz@uptc.edu.co
}

Recibido: 18 de enero de 2019

Aceptado para publicación: 12 de septiembre de 2019 
of the first group were supplemented with orange/oat silage and in the second with only oatmeal silage for 30 days. The animals maintained their metabolic status, body condition and milk production. Cholesterol was the only parameter found higher in animals supplemented with orange/oat silage as compared to the animals fed with only oatmeal silage. It is concluded that both types of silage for supplementation is equivalent, but the acceptance by the animals of the orange/oat silage was notoriously superior.

Key words: bovine; silage; metabolism; nutrition; feeds

\section{INTRODUCCIÓN}

Las condiciones medioambientales del trópico altoandino colombiano representan un desafío para la producción de leche bovina, no solo por los aspectos genéticos del ganado especializado (baja rusticidad), sino también por aquellos de orden sanitario, climático y nutricional (Holman et al., 2003). El 70\% de la producción de leche del país proviene de zonas sobre los $2000 \mathrm{msnm}$ (trópico altoandino), donde los animales se exponen a temperaturas bajas en las madrugadas y de temperaturas altas durante el día, por lo que el gasto energético para lograr una regulación adaptativa puede propiciar una menor producción de leche (Carulla y Ortega, 2016; DANE, 2016). Las razas lecheras sufren estrés térmico cuando se exponen a temperaturas fuera del rango de confort y más aún cuando ocurren en corto tiempo, lo que repercute en una baja producción de leche, ya que el consumo de materia seca puede disminuir (Campos et al., 2007; DANE, 2016).

En la Granja Experimental Tungüavita (GET), así como en otras ganaderías de la zona, se presentan un déficit de forraje en el verano que no permite cumplir con los requerimientos nutricionales de los animales. El suplemento con concentrado permite corregir estas deficiencias, pero aumenta los costos de producción, conduciendo a la necesidad de utilizar alternativas nutricionales más económicas. Los subproductos agroindustriales y los desechos de cosecha constitu- yen una alternativa para la alimentación del ganado a un menor costo que el alimento concentrado, entre los cuales se encuentran los residuos de los cítricos (jugos, pulpa y derivados) (Martínez et al., 2008; BermúdezLoaiza et al., 2015).

La cáscara de naranja, con base en materia seca, contiene $27.3 \mathrm{mg} / 100 \mathrm{~g}$ de calcio, $8.64 \mathrm{mg} / 100 \mathrm{~g}$ de magnesio, $0.38 \mathrm{mg} / 100$ $\mathrm{g}$ de zinc, $16.25 \mathrm{mg} / 100 \mathrm{~g}$ de ácido ascórbico y $2.25 \mathrm{mg} / 100 \mathrm{~g}$ de carotenoides totales, así como 43.3 g EAG (equivalente ácido gálico)/ $\mathrm{kg}$ de polifenoles totales (Rincón et al., 2005). Dentro de esos fenoles se destacan los flavonoides y ácidos fenólicos, los cuales tienen una capacidad antioxidante demostrada (Blanco et al., 2005; Rincón et al., 2005). Los mamíferos sintetizan L-ácido ascórbico en el hígado; sin embargo, su consumo en la dieta representa un valioso aporte, de allí que los cítricos constituyen uno de los principales componentes de la ración en algunos animales (Smirnoff, 2018). Los rumiantes, en cambio, dependen de la síntesis endógena de este, ya que el aporte en la dieta es destruido por los microorganismos ruminales.

En general, la síntesis de vitamina $\mathrm{C}$ en el rumiante es suficiente para el mantenimiento de sus funciones sin necesitar de suplementación adicional; sin embargo, algunos autores indican que los bovinos en condiciones de enfermedad o de stress podrían requerir un suministro adicional de la vitamina (Ranjan et al., 2012), mientras que otros indican que la síntesis endógena es suficiente, incluso en 
bovinos en alta producción, así como en situaciones como la cetosis (Santos et al., 2001; Padilla et al., 2006; Ranjan et al., 2012). Por lo anterior, y frente a la necesidad de buscar alternativas alimenticias en los bovinos, el presente estudio tuvo como objetivo determinar el efecto de la suplementación con ensilaje de cáscara de naranja/avena en algunos parámetros metabólicos de vacas y de la calidad de la leche producida en época de verano.

\section{Materiales y Métodos}

El estudio fue de tipo experimental y se realizó en la Granja Experimental Tungüavita, de propiedad de la Universidad Pedagógica y Tecnológica de Colombia, ubicada en el municipio de Paipa, departamento de Boyacá (Colombia). La granja se encuentra sobre la cordillera oriental colombiana, a $2525 \mathrm{msnm}$, y presenta una temperatura promedio anual de $13-14.4{ }^{\circ} \mathrm{C}$, con una época de verano (temperaturas hasta $22{ }^{\circ} \mathrm{C}$, con sequías y heladas en las madrugadas) en los meses de diciembre, enero y febrero.

\section{Animales de Estudio}

Se utilizaron 20 vacas adultas lactantes Holstein, Jersey, Simmental y sus cruces, de segundo y tercer parto, con condición corporal 3.5 y con gestaciones entre 2 y 4 meses, de manera que al culminar el estudio no excedieran de los 6 meses de gestación. Las vacas se mantienen en pastoreo rotacional en praderas de pasto kikuyo (Cenchrus clandestinus), sal y agua a voluntad. Durante el ordeño, se administra concentrado proporcional a la producción $(1-2 \mathrm{~kg})$. Se realizó un examen clínico completo a las vacas, encontrándolas en aparente buen estado de salud. Los animales estaban vacunados contra la fiebre aftosa, brucelosis y fueron desparasitados con albendazol; además, se les hacía controles periódicos contra la mosca. Durante el estudio, los animales se mantuvieron en potreros separados, según el grupo de tratamiento, y bajo las mismas condiciones de pastura.
Los animales seleccionados, con base a la condición corporal, etapa de lactancia, número de partos y peso corporal, fueron divididos al azar en dos grupos: tratado $(\mathrm{n}=10)$, suplementado con ensilaje de naranja y avena; control $(\mathrm{n}=10)$, suplementado solo con ensilaje de avena. Cada animal constituyó una unidad experimental. Las vacas tuvieron 15 días de acostumbramiento a la nueva ración y luego 30 días en la fase experimental, lo que permitió el consumo de la nueva ración por 40 días aproximadamente. El estudio se realizó entre los meses de diciembre y febrero.

\section{Variables}

De acuerdo con el diseño metodológico, se manejó como variable independiente el tipo de ensilaje (naranja/avena o solo avena), quedando controladas las demás posibles variables. Como variables dependientes se consideraron valores de creatinina, colesterol, triglicéridos, urea, calcio, así como los niveles de las enzimas gamma glutamil transferasa (GGT), alanina aminotransferasa (ALT) y aspartato aminotransferasa (AST) y de las hormonas triyodotironina $\left(\mathrm{T}_{3}\right)$ y tiroxina $\left(\mathrm{T}_{4}\right)$. Así mismo, se consideraron los parámetros propios de la calidad composicional de la leche para determinar posibles variaciones por efecto de las dietas.

\section{Procedimientos}

Para la preparación del ensilaje se usó avena con una edad vegetativa de 140 días. La avena fue cortada con una cosechadora obteniéndose cortes de 4-5 cm. Los materiales (avena y naranja) fueron premarchitados por 6 horas (Figura 1). La naranja se usó en mitades ( $8-9 \mathrm{~cm}$ de diámetro y $0.5-0.8 \mathrm{~cm}$ de grosor) con presencia de muy pocas semillas. El ensilaje de avena con cáscara de naranja se hizo en una proporción de 70:30, respectivamente; proporción establecida con base a estudios previos, no publicados, que permiten obtener un ensilaje estable en calidad composicional. En los dos tipos de ensilaje se usaron bacterias ácido-lácticas (BAL) como inoculantes en proporción de $2 \%$. El 

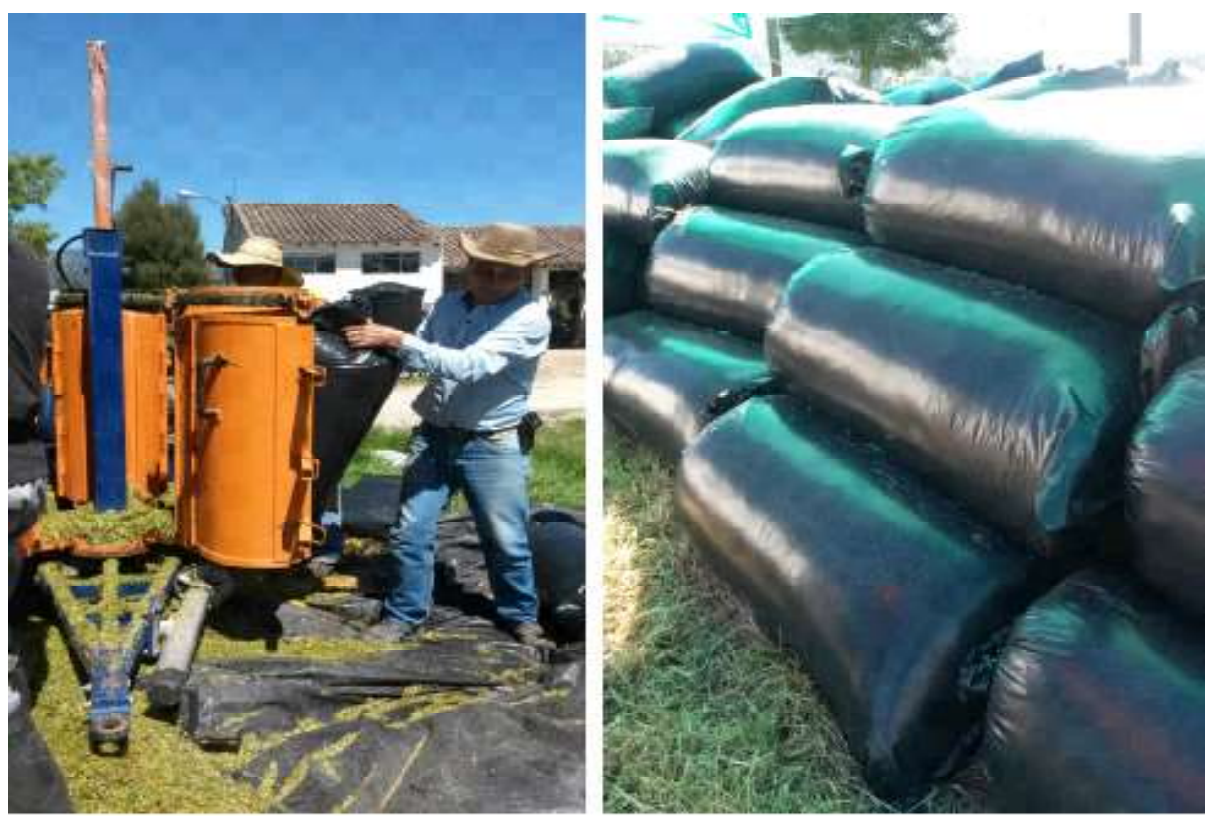

Figura 1. Elaboración y almacenamiento del ensilaje de naranja/avena y de avena

ensilado fue empacado y compactado en bolsas con capacidad para $50 \mathrm{~kg}$ y fermentado por 25 días (Figura 2). El tiempo que transcurrió entre la salida de la cáscara de la planta industrial y su empaque para el ensilaje fue de 6 horas, tiempo considerado de premarchitamiento que fue similar el tiempo transcurrido para la avena desde el corte.

La administración del suplemento se hizo diariamente colocando el ensilaje en el potrero correspondiente a cada grupo, proporcionándose $2 \mathrm{~kg} / \mathrm{animal}$, en base seca. La cáscara de naranja tuvo $75 \%$ de humedad y $8 \%$ de proteína cruda con base en la materia seca. Dos muestras tomadas por triplicado de cada ensilaje se enviaron al Laboratorio de Nutrición Animal de la Universidad Pedagógica y Tecnológica de Colombia (UPTC). Una muestra se utilizó para el análisis bromatológico y la otra muestra fue secada y remitida al Laboratorio de Nutrición Animal de la Universidad Nacional de Colombia, para determinar la digestibilidad in vitro.
Se tomaron muestras de sangre $(4-5 \mathrm{ml})$, la primera antes de iniciar la administración de los tratamientos y la segunda a los 30 días del inicio de la fase experimental. Las muestras de sangre fueron centrifugadas a 2500 rpm por 5 minutos, se extrajo el suero y se llevó refrigerado en un tiempo no mayor a 2 horas al laboratorio «Animal-Lab» en la ciudad de Sogamoso (Boyacá, Colombia). El laboratorio utilizó un equipo MegaQuant${ }^{\mathrm{T}}$ Wave, que posee un sistema de fotómetro biocromático con microproce-sador, con el cual se miden absorbancias o concentraciones basadas en los puntos standard. La leche de cada animal del ordeño de la tarde fue homogenizada y se tomaron por duplicado, llevándose en recipientes refrigerados al laboratorio donde fueron procesadas en un analizador lácteo MilkoScan ${ }^{\mathrm{TM}}$ Mars (Foss).

Se estimó el costo del ensilaje para determinar si permitía ser considerado como alternativa rentable para la suplementación de los animales. 


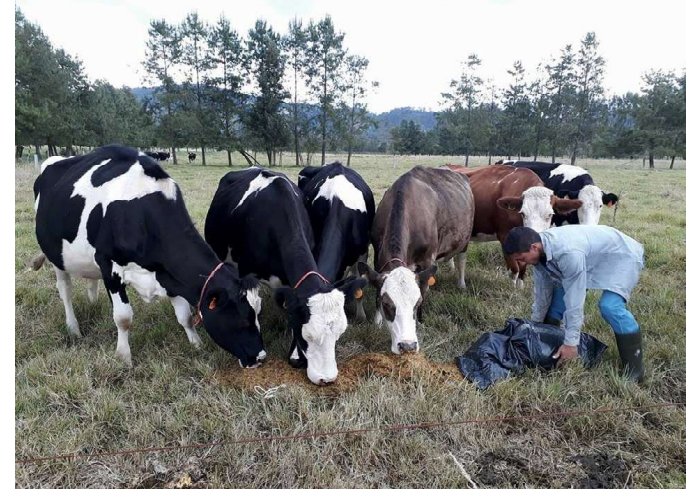

Figura 2. Suministro de los ensilajes a los animales de estudio

\section{Análisis Estadístico}

La evaluación del efecto del tipo de suplementación sobre el metabolismo de los animales y sobre la calidad composicional de la leche se hizo mediante un análisis de varianza cuya variable independiente fue el tipo de ensilaje. Los datos se sometieron a supuestos de normalidad a través del estadístico de Shapiro-Wilk y homogeneidad por prueba de Levene. Para las variables que cumplieron los supuestos se sometieron a la prueba «t» Student con un nivel de confianza del $95 \%$. El paquete estadístico usado fue SPSS v. 19.

\section{Resultados}

El ensilaje de naranja/avena fue aceptado por todos los animales del grupo tratado desde el primer día de su administración; en cambio, la aceptación del ensilaje de avena por parte de los animales del grupo control fue menor, tomando cinco días para que todos lo consumieran. En ambos casos se respetó el tiempo de acostumbramiento que se había planteado. Los animales mantuvieron su condición corporal y no se observaron signos de enfermedad.

La composición de los dos ensilajes fue similar, encontrándose valores análogos para materia seca, proteína cruda y extracto etéreo; sin embargo, los porcentajes de fibra detergente neutra y ácida fueron superiores en el ensilaje de naranja/avena. Todos estos datos presentaron una distribución normal (Shapiro-Wilk) y homogeneidad de las varianzas (Est-Levene). Por su parte, la digestibilidad in vitro del ensilaje de avena fue de $66.1 \%$, mientras que del ensilaje de naranja/avena fue de $63.7 \%$ (Cuadro 1 ; $\mathrm{p}<0.05$ ).

Los parámetros metabólicos de las vacas de ambos grupos de suplementación se encontraban dentro de los rangos normales, no habiendo diferencias estadísticas entre los parámetros valorados, excepto en el colesterol, cuyo valor fue superior en los animales que recibieron el ensilaje de naranja/ avena $(\mathrm{p}<0.05)$. Las concentraciones de las enzimas hepáticas estuvieron dentro de lo normal para la GGT y por encima de estos para AST y ALT (Cuadro 2).

Los parámetros composicionales de la leche estuvieron dentro de los rangos esperados y sin diferencias estadísticas entre gru-

Cuadro 1. Calidad composicional de ensilaje de avena y ensilaje de avena-naranja

\begin{tabular}{lccccccc}
\hline & \% MS & \% PC & \% EE & \% CZ & \% FDN & \% FDA & \% DIVMS \\
\hline $\begin{array}{l}\text { Ensilaje de } \\
\text { avena }\end{array}$ & 31 & 7.2 & 6.8 & 6.7 & 62.4 & 40.0 & 66.1 \\
$\begin{array}{l}\text { Ensilaje de } \\
\text { naranja/avena }\end{array}$ & 30 & 7.2 & 6.0 & 5.7 & 64.7 & 48.4 & 63.7 \\
\hline
\end{tabular}

MS: materia seca; PC: proteína cruda; EE: extracto etéreo; CZ: cenizas; FDN: fibra detergente neutra; FDA: fibra detergente ácida; DIVMS; digestibilidad in vitro de la MS 
Cuadro 2. Química sanguínea (promedio \pm desviación estándar) en muestras de sangre de vacas suplementadas con ensilaje de naranja/avena y con solo ensilaje de avena

\begin{tabular}{lccc}
\hline & $\begin{array}{c}\text { Con ensilaje de } \\
\text { naranja/avena }\end{array}$ & $\begin{array}{c}\text { Con ensilaje de } \\
\text { avena }\end{array}$ & ${\text { Rango } \text { normal }^{1}}^{{ }^{2}}$ \\
\hline Creatinina, $\mathrm{mg} / \mathrm{dl}$ & $0.98 \pm 0.34$ & $1.01 \pm 0.31$ & $1-2$ \\
Colesterol, mg/dl & $202.7 \pm 25.3$ & $176.8 \pm 16.2$ & $175-235$ \\
Triglicéridos, mmol/1 & $0.46 \pm 0.28$ & $0.41 \pm 0.22$ & $0.1-0.49$ \\
Urea, mmol/1 & $5.78 \pm 1.62$ & $5.31 \pm 1.75$ & $2.6-7$ \\
Calcio, mg/dl & $10.88 \pm 0.8$ & $10.37 \pm 1.1$ & $9.7-12.4$ \\
ALT, U/l & $66.88 \pm 7.7$ & $67.72 \pm 10.1$ & $14-38$ \\
$\mathrm{AST}, \mathrm{U} / \mathrm{l}$ & $158.7 \pm 33.3$ & $159.0 \pm 19.6$ & $78-132$ \\
$\mathrm{GGT}, \mathrm{U} / 1$ & $9.7 \pm 2.1$ & $11.2 \pm 1.9$ & $7.8-17$ \\
$\mathrm{~T}_{3}, \mathrm{ng} / \mathrm{dl}$ & $3.76 \pm 1.74$ & $4.69 \pm 2.52$ & $2.3-4.4$ \\
$\mathrm{~T}_{4}, \mathrm{nmol} / \mathrm{l}$ & $1.62 \pm 0.93$ & $1.96 \pm 1.01$ & $1.9-3.9$ \\
\hline
\end{tabular}

${ }^{1}$ Valores normales reportados por el laboratorio "Animal-Lab" (Boyacá, Colombia)

Cuadro 3. Calidad composicional y recuento de células somáticas obtenidos de vacas suplementadas con ensilaje de naranja/avena y solo de avena (10 vacas por grupo)

\begin{tabular}{lcc}
\hline & $\begin{array}{c}\text { Con ensilaje } \\
\text { de naranja / } \\
\text { avena }\end{array}$ & $\begin{array}{c}\text { Con } \\
\text { ensilaje de } \\
\text { avena }\end{array}$ \\
\hline $\mathrm{pH}$ & $6.8 \pm 0.12$ & $6.7 \pm 0.18$ \\
Grasa (\%) & $4.0 \pm 0.73$ & $3.8 \pm 0.68$ \\
Densidad & $25.0 \pm 0.64$ & $25.4 \pm 1.01$ \\
Sólidos no & $7.3 \pm 0.3$ & $7.3 \pm 0.49$ \\
grasos & $2.2 \pm 0.23$ & $2.3 \pm 0.26$ \\
Proteína (\%) & $0.6 \pm 0.05$ & $0.6 \pm 0.03$ \\
Sales (\%) & $4.4 \pm 0.48$ & $4.4 \pm 0.35$ \\
Lactosa (\%) & & \\
Células & & $90 \pm 0$ \\
somáticas & $90 \pm 0$ & \\
(RCSx10 & & \\
\hline
\end{tabular}

pos. Así mismo, el recuento de células somáticas y el porcentaje de sales, lactosa y de sólidos no grasos fueron estadísticamente similares entre ambos grupos (Cuadro 3).
El ensilaje de avena bajo las condiciones de este trabajo tuvo un costo de $\$ 257$ (pesos colombianos - COP) por kilogramo, que equivale aproximadamente a US\$ 0.09). Por su parte, el ensilaje de avena/naranja en la proporción 70:30 utilizado en el estudio tuvo un costo de COP $\$ 220 / \mathrm{kg}$ (US\$ 0.06).

\section{Discusión}

Se observó una alta palatabilidad del ensilaje de naranja/avena, manifestada por su aceptación inmediata en la dieta, tal como lo describe la literatura (Benitezy Poveda, 2011), lo cual junto a la facilidad de su elaboración y la necesidad de buscar alternativas de alimentación en épocas de verano, permiten asegurar que podría constituir una alternativa económica para mantener el estado metabólico normal de las vacas en producción ante una baja de oferta de forraje.

Mojica et al. (2009) reporta valores que van desde el $58.1 \%$ para la DIVMS; sin embargo, se indica que la digestibilidad se relaciona directamente con la edad vegetativa de 
la planta, habiendo $74.1 \%$ de DIVMS en corte temprano, reduciéndose a $52.3 \%$ a una edad vegetativa mayor (Dumont et al., 2005). En el caso de ensilajes mixtos de avena con otros forrajes, se reporta una DIVMS de 67.1\%, señalando que un aumento de la digestibilidad se asocia con una reducción en el tiempo de colonización de las bacterias fermentativas e infiriendo en una menor FDA (Blanco et al., 2005).

Los valores de colesterol se encuentran por debajo de los valores de referencia, cuando el animal se encuentra en un balance energético negativo (Reist et al., 2002; Campos et al., 2007). En este estudio, las vacas suplementadas con los dos tipos de ensilaje presentaron valores de colesterol dentro de los valores de referencia (Campos et al., 2007; Faye et al., 2015). Algunos estudios permiten afirmar que la fuente de grasa en la dieta influye en los niveles sanguíneos de colesterol y en la leche; en este sentido, el suministro de ensilaje aumenta la concentración de colesterol respecto a una dieta basada en forraje (Reklewska et al., 2002; Barlowska et al., 2011).

En este estudio los valores de creatinina ( 0.98 y 1.01 para el grupo tratado y el grupo control, respectivamente), estuvieron dentro de los rangos de referencia (Reist et al., 2002). Los niveles de creatinina si bien son indicativos de la función renal (Issi et al., 2016), también se sabe que disminuyen en animales de bajo peso y que aumentan cuando por alguna razón, se desencadena el catabolismo proteico en el animal (Issi et al., 2016), lo que indica que los animales de este estudio mantuvieron su balance de nitrógeno dentro de lo normal.

En los animales de estudio, los valores de urea estuvieron dentro de lo normal, lo que indica que la dieta no los alteró y que reflejan un adecuado funcionamiento renal. El aumento de los valores de nitrógeno ureico o de urea aumentan ante una falla renal o por un excesivo consumo de proteína bruta en la dieta (Campos et al., 2007). Los niveles de nitró- geno ureico en el plasma se han correlacionado con aspectos reproductivos, ya que sus altas concentraciones coinciden con altos niveles de nitrógeno ureico y de amoniaco en el fluido folicular durante el estro y en el fluido uterino durante la fase luteal, lo cual causa embriotoxicidad (Hammon et al., 2005). La suplementación del estudio no afectó los niveles de nitrógeno ureico, disminuyendo el riesgo de alteraciones reproductivas debidas a posibles excesos.

Se sabe que la concentración de glucosa sérica disminuye al comenzar la lactancia por efecto del balance energético negativo debido al inicio de la producción láctea y al menor consumo de alimento (Steen, 2001). En el presente estudio, no obstante, las vacas presentaron valores de glucosa dentro de lo esperado, que según Campos et al. (2007) son de $2.9 \mathrm{nmol} / 1$ en Holstein, $3.2 \mathrm{nmol} / 1 \mathrm{en}$ Jersey y $2.6 \mathrm{nmol} / 1$ en Simmental.

El perfil metabólico es indicativo del desempeño metabólico fisiológico de los animales. Según Campos et al. (2007), el nivel de ALT en vacas Holstein, Jersey y Simmental es de $26.5,35.9$ y 32.8 U/1, respectivamente, mientras que en los animales de este estudio estuvieron en 66.9 y $66.8 \mathrm{U} / 1$ en los animales suplementados con ensilaje de naranja/avena y solo avena, respectivamente. En forma similar, Campos et al. (2007) reportan valores de AST y GCT de $65.5 \mathrm{y}$ 17.6 U/1 en vacas Holstein, 57.4 y $7.8 \mathrm{U} / 1$ en Jersey y de 65.5 y 10.1 U/1 en Simmental, siendo estos valores superiores al inicio y al final de la lactancia, mientras que en los animales de este estudio estuvieron en $158.7 \mathrm{y}$ 9.7 en los animales suplementados con ensilaje de naranja/avena, respectivamente y de 159.0 y $11.2 \mathrm{U} / 1$ en los animales suplementados con ensilaje de solo avena, respectivamente, en todos los casos dentro de los valores esperados. Los rangos reportados para estas enzimas son variados y se encuentran algunos más amplios que los indicados anteriormente para AST (90-226 U/L) (Villa et al., 1999), lo que dificulta en muchos casos la interpretación de los resultados. 
La alta actividad del aspartato aminotransferasa (AST) y de la gamma glutamiltransferasa (GGT), se asocian con el síndrome de hígado graso, disminución del apetito o cetosis en lactancia temprana (Steen, 2001; Stojeviæ et al., 2005). Todos los animales de este estudio presentaron concentraciones aumentadas de AST y ALT respecto a los valores normales (Villa et al., 1999; Campos et al., 2007; Issi et al., 2016); sin embargo, no se observaron signos de enfermedad. Por otro lado, los valores de las hormonas tiroideas se mantuvieron dentro de los valores normales para la especie (Osorio et al., 2014; Djokovic et al., 2010), lo cual indica no solo el adecuado funcionamiento del eje hipotálamo-hipófisis-tiroides, sino además, el balance metabólico que mantuvieron los animales durante la época de verano.

La administración de concentrado o de cereales de fácil fermentación aumenta la producción de ácido láctico y, por ello, disminuyen el pH ruminal, propiciando el desarrollo de acidosis ruminal. A pesar de que en este estudio no se midió el pH ruminal, los animales no mostraron sintomatología de acidosis ruminal o indigestión. En ovinos se sabe de los beneficios de la inclusión de productos cítricos en proporción inferior al 30\% de la ración, como se hizo en este estudio, no afecta la fisiología ruminal (Piquer et al., 2009).

Los parámetros de la calidad composicional de la leche de las vacas suplementadas con los dos tipos de ensilaje (Cuadro 2) mostraron un comportamiento similar a los parámetros que se consideran normales para las razas que hicieron parte del estudio (Benitez y Poveda, 2011). Solo el porcentaje de grasa en leche de las vacas que recibieron ensilaje de avena/naranja fue superior al de aquellas con solo avena. La inclusión de productos cítricos en la ración de vacas en producción promueve la producción de ácido acético y, por ello, un aumento en la producción de grasa en la leche (Piquer et al., 2009). El porcentaje de proteína fue similar en am- bos grupos experimentales, a diferencia del reporte de Benitez y Poveda (2011), quienes encontraron menor nivel de proteína en vacas suplementadas con ensilaje de naranja que en aquellas que recibieron cereales. Por otro lado, el $\mathrm{pH}$ esperado de la leche es de 6.5-6.8, ligeramente ácido, debido a la presencia de proteínas y aniones fosfóricos; valores que no difieren con los obtenidos en el presente estudio y con los reportados por Bermúdez-Loaiza et al. (2015).

El menor costo de producción del ensilaje de cáscara de naranja con avena es muy favorable para la rentabilidad del sistema de producción lechera de la granja del estudio o de cualquier otra que opte por un ensilaje de este tipo. No obstante, se debe tener en cuenta que el costo de la avena en la región depende de la época del año, aumentando de precio durante el verano.

\section{Conclusiones}

- El ensilaje de naranja/avena y de solo avena para la suplementación de bovinos de leche son equivalentes, pero la aceptación por parte de los animales del ensilaje de naranja/avena fue notoriamente superior.

- $\quad$ El ensilaje de naranja/avena fue de alta palatabilidad, y con alta concentración de fibra detergente neutra y ácida con relación al ensilaje de solo avena.

- Los animales se mantuvieron metabólicamente estables durante el verano.

\section{Agradecimientos}

Los autores del trabajo expresan su agradecimiento a la Vicerrectoría de Investigaciones y Extensión de la Universidad Pedagógica y Tecnológica de Colombia por la financiación del proyecto y al personal de la Granja Experimental Tungüavita por facilitar la realización del estudio; así mismo a los estudiantes Maycolt Alexis Niño y Luis Alejandro Rodríguez por la colaboración prestada. 


\section{Literatura Citada}

1. Barlowska J, Szwajkowska M, Litwinczuk Z, Matwijczuk A. 2011. The influence of cow breed and feeding system on the dispersion state of milk fat and content of cholesterol. Annals Polish Assoc Zootech 7: 57-65

2. Benitez SY, Poveda CA. 2011. Evaluación nutricional de ensilajes con diferentes niveles de inclusión de cáscara de naranja (Citrus sinensis) y digestibilidad in vivo como alternativa energética para alimentación de cerdos. Rev Colomb Cienc Anim 4: 20-28. doi: 10.24188/recia

3. Bermúdez-Loaiza JA, Melo-Camacho EP, Estrada-Álvarez J. 2015. Evaluación de ensilaje de naranja entera (Citrus sinensis) como alternativa de suplementación en bovinos. Vet Zootec 9: 38-53. doi: 10.17151/vetzo.2015.9.2.4

4. Blanco GM, Chamorro DR, Arreaza LC, Rey AM. 2005. Evaluación nutricional del ensilaje de Sambucus peruviano, Acacia decurrens y Avena sativa. CORPOICA 6: 81-85. doi: 10.21930/rcta

5. Campos GR, Cubillos C, Rodas A. 2007. Indicadores metabólicos en razas lecheras especializadas en condiciones tropicales en Colombia. Acta Agron 56: 85-92. doi: 10.15446/acag

6. Carulla JE, Ortega E. 2016. Sistema de producción lechera en Colombia: retos y oportunidades. Arch Latinoam Prod Anim 24: 83-87.

7. [DANE] Departamento Administrativo Nacional de Estadística. 2016. Efecto del clima en la ganadería de leche. Boletín mensual. Insumos y factores asociados a la producción agropecuaria. No. 45. [Internet]. Disponible en https://www.dane.gov.co/files/investigaciones/agropecuario/sipsa/Bol_Insumos_mar_2016.pdf

8. Djokoviae R, Šamanc H, Bojkovski J, Fratria N. 2010. Blood concentrations of thyroid hormones and lipids of dairy cows in transitional period. Acta Vet Brno
43: 43-40. doi: 10.2754/avb200776040525

9. Dumont J, Anrique ERG, Alomar D. 2005. Efecto de dos sistemas de determinación de materia seca en la composición química y calidad del ensilaje directo de avena en diferentes estados fenológicos. Agric Téc 65: 388-396. doi: 10.4067/S0365-28072005000400005

10. Faye B, Bengoumi M, Al-Masaud A, Konuspayeva G. 2015. Comparative milk and serum cholesterol content in dairy cow and camel. J King Saud Univ Sci 27: 168-175. doi: 10.1016/j.jksus.2014.11.003

11. Hammon DS, Holyoak GR, Dhiman TR. 2005. Association between blood plasma urea nitrogen levels and reproductive fluid urea nitrogen and ammonia concentrations in early lactation dairy cows. Anim Reprod Sci 86: 195-204. doi: 10.1016/j.anireprosci.2004.08.003

12. Holmann F, Rivas L, Carulla J, Giraldo LA, Guzmán S, Martínez M, Rivera B, et al. 2003. Evolución de los sistemas de producción de leche en el trópico latinoamericano y su interrelación con los mercados: un análisis del caso colombiano. Cali, Colombia: CIAT-Consorcio Tropileche. $53 \mathrm{p}$.

13. Issi M, Gül Y, Basbug O. 2016. Evaluation of renal and hepatic functions in cattle with subclinical and clinical ketosis. Turk J Vet Anim Sci. 40: 47-52. doi: 10.3906/vet-1505-16

14. Martínez MJ, Chongo GB, Vázquez $J H$, Hernández $S N$, Fontes MD, Lezcano MY, Cubillas LN. 2008. Características nutritivas de los hollejos húmedos de naranja (Citrus sinensis cv. Valencia) mantenidos en estibas. Tec Pecu Mex 46: 183-193.

15. Mojica RJE, Castro RE, León CJ M. Cárdenas REA, Pabón RM, Carulla FJE. 2009. Efecto de la oferta de pasto kikuyo y ensilaje de avena sobre la producción y calidad composicional de la leche bovina. Corpoica 10: 81-90. 
16. Osorio JH, Vinasco RJ, Suárez YJ. 2014. Hormonas tiroideas en bovinos: artículo de revisión. Biosalud 13: 76-84.

17. Padilla L, Matsui T, Kamiya Y, Kamiya M, Tanaka M, Yano H. 2006. Heat stress decreases plasma vitamin $\mathrm{C}$ concentration in lactating cows. Livest Sci 101: 300-304. doi: 10.1016/j.livprodsci.2005.12.002

18. Piquer O, Ródenas L, Casado C, Blas E, Pascual JJ. 2009. Whole citrus fruits as an alternative to wheat grain or citrus pulp in sheep diet: effect on the evolution of ruminal parameters. Small Ruminant Res 83: 14-21. doi: 10.1016/ j.smallrumres.2008.11.009

19. Ranjan R, Ranjan A, Dhaliwal GS, Patra RC. 2012. L-Ascorbic acid (vitamin C) supplementation to optimize health and reproduction in cattle. Vet Quart 32: 145-150. doi: 10.1080/ 01652176.2012 .734640

20. Reist M, Erdin DK, VonEuw D, Tschümperlin KM, Leuenberger $H$, Hammon HM, Morel C, et al. 2002. Postpartum reproductive function: association with energy, metabolic and endocrine status in high yielding dairy cows. Theriogenology 59: 1707-1723. doi: 10.1016/S0093-691X(02)01238-4

21. Reklewska B, Oprzadek A, Reklewski Z, Panicke L, Kuczynska B, Oprzadek $J$. 2002. Alternative for modifying the fatty acid composition and decreasing level in the milk of cows. Livest Prod Sci 76: 235-243. doi: 10.1016/S03016226(02)00123-9
22. Rincón MA, Vásquez AM, Padilla FC. 2005. Composición química y compuestos bioactivos de las harinas de cáscaras de naranja (Citrus sinensis), mandarina (Citrus reticulata) y toronja (Citrus paradisi) cultivadas en Venezuela. Arch Latinoam Nutr 55: 305-310.

23. Santos MV, Lima FR, Rodrigues PHM, Barros SBM, Laranja Fonseca LFL. 2001. Plasma ascorbate concentrations are not correlated with milk somatic cell count and metabolic profile in lactating and dry cows. J Dairy Sci 84: 134-139. doi: 10.3168/jds.S00220302(01)74461-X

24. Smirnoff N. 2018. Ascorbic acid metabolism and functions: a comparison of plants and mammals. Free Radic Biol Med 122: 116-129. doi: 10.1016/j.freeradbiomed.2018.03.033

25. Steen A. 2001. Field study of dairy cows with reduced appetite in early lactation: clinical examinations, blood and rumen fluid analyses. Acta Vet Scand 42: 219228. doi: 10.1186/1751-0147-42-219

26. Stojeviae Z, Piršljin J, Milinkoviae-Tur S, Zdelar-Tuk M, Ljubice BB. 2005. Activities of AST, ALT and GGT in clinically healthy dairy cows during lactation and in the dry period. Vet Arhiv 75: 67-73.

27. Villa NA, Ceballos A, Cerón D, Serna $C A$. 1999. Valores bioquímicos sanguíneos en hembras Brahman bajo condiciones de pastoreo. Pesq Agropec Bras 34: 2339-2343. 